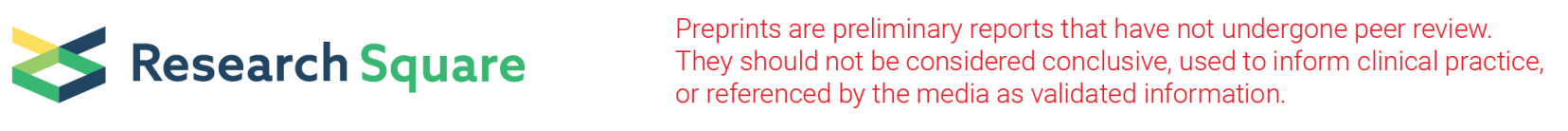

\title{
Association between eNOS rs1799983 Polymorphism and Hypertension: a Meta-analysis involving 14,185 Cases and 13,407 Controls
}

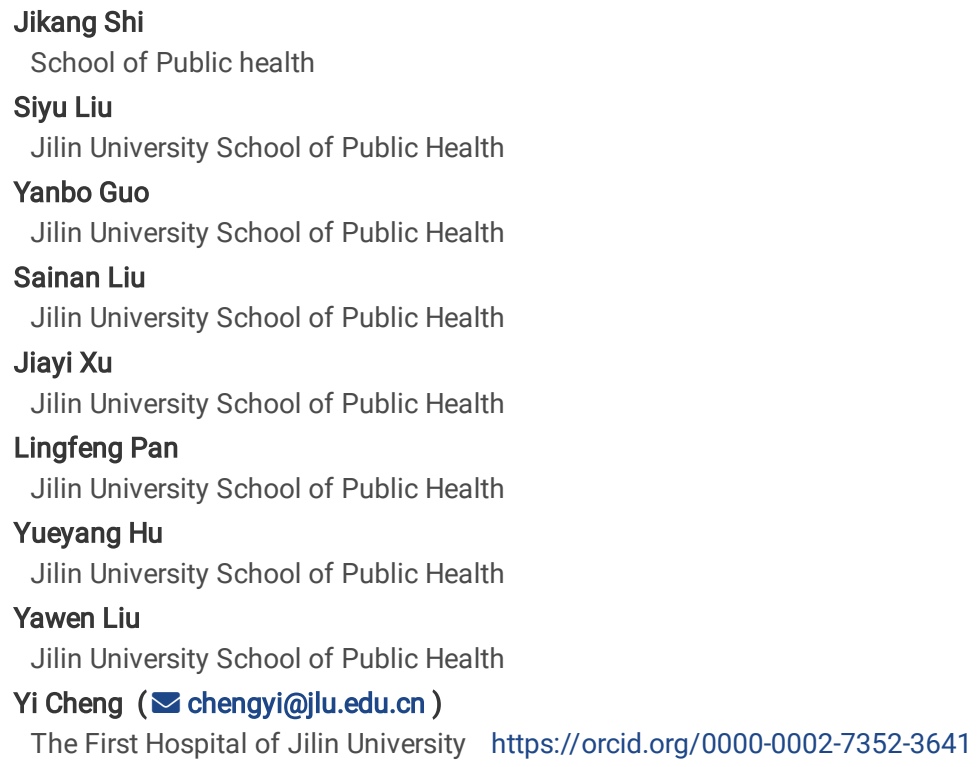




\section{Abstract}

Background: Essential hypertension (EH) is a complex disease determined by the interaction of genetic and environmental factors, eNOS is considered to be one of the susceptible genes for hypertension. Our study aimed to evaluate the association between eNOS rs 1799983 polymorphism and hypertension, and to provide evidence for the etiology of hypertension.

Methods: Case-control studies of eNOS rs1799983 polymorphism and hypertension were included by searching PubMed, Embase, Web of Science, Medline, Scopus, WanFang datebase, Vip datebase, and CNKI database. Eligible data were extracted and pooled, and were analyzed using R software based on five different genetic models.

Results: A total of 60 eligible articles involving 14,185 cases and 13,407 controls were finally selected. We found significant association between eNOS rs1799983 polymorphism and hypertension under any genetic model (T vs G: $O R=1.44,95 \% C l=1.26-1.63$; GT vs GG: $O R=1.34,95 \% C l=1.18-1.52$; TT vs GG: $O R=1.80,95 \% C l=1.41-2.31 ; \mathrm{GT}+\mathrm{TT}$ vs GG: $O R=1.42,95 \% C l=1.25-1.63 ; \mathrm{TT}$ vs GG+GT: $O R=1.68,95 \% C l=1.35-2.08 ; \mathrm{GT}$ vs GG+TT: $O R=1.24,95 \% C l=1.11-1.40)$

Conclusions: We found that eNOS rs1799983 polymorphism is associated with the increased risk of hypertension under any genetic model. Moreover, investigations of gene-gene and gene-environment interactions are needed to give more insight into the association between eNOS rs 1799983 polymorphism and hypertension.

\section{Background}

Essential hypertension (EH) is a complex disease determined by the interaction of genetic and environmental factors, and EH is regarded as a predisposing risk factor for many diseases, such as myocardial infarction, stroke, and chronic renal failure [1]. So far, the exact pathogenesis of hypertension is still unclear in spite of the in-depth research. However, more and more evidence supports genetic factors to a large extent cause hypertension [2], thus it is essential to identify susceptible genes for prevention, diagnosis, and treatment of hypertension [3]. Gene (eNOS) encoding endothelial nitric oxide synthase is considered to be one of the susceptible genes for hypertension because its enhanced production or enzyme bioavailability can lead to constitutive release of nitric oxide (NO) in endothelial cells, which is involved in blood pressure (BP) regulation [4].

Previous studies have shown that eNOS plays a critical role in regulating vascular tone and blood pressure. For example, overexpression of eNOS gene in transgenic mice leads to a significant decrease in blood pressure [5]. In addition, it was found that inhibition of eNOSgene in healthy individuals is associated with decreased levels of NO release and increases blood pressure [6].

The eNOS gene at 7q35-36 spans $21 \mathrm{~kb}$, with 26 exons and 25 introns. There are about 10 polymorphic loci distributed in the promoter, exon, and intron of the eNOS gene. In these loci, the common mutation that leads to amino acid substitutions in mature proteins is G894T or Glu298Asp (rs1799983) mutations, in which base substitution of $\mathrm{G}$ to $\mathrm{T}$ will result in glutamic acid (Glu) being replaced at exon 7 by aspartic acid (Asp) at position 298 of the corresponding amino acid [7]. This genetic mutation reduces the production of NO and subsequently affects the development of EH [8].

A large number of articles have studied the association between eNOS rs1799983 polymorphism and hypertension; however, these results are still contradictory. Recently, it is worth noting that new studies [9-12] on this theme have been published since the last meta-analysis published in 2017 [13]. Therefore, we included these newly published studies and conducted a further meta-analysis to investigate whether eNOS rs1799983 polymorphism is associated with hypertension.

\section{Materials And Methods}

\section{Literature search strategy}

Systematic literature search was performed in PubMed, Embase, Web of Science, Medline, Scopus, WanFang datebase, Vip datebase, and CNKI database up to October 30, 2020. Various combinations of terms used for searching were ("endothelial nitric oxide synthase" OR "nitric oxide synthase type III" OR "eNOS" OR “NOS3") AND ("polymorphism" OR "variant" OR “mutation”) AND ("hypertension” OR "high blood pressure”). Moreover, we also retrieved and scrutinized related articles from the reference lists of literatures to replenish literatures that had not been identified in the initial search.

\section{Inclusion/exclusion criteria}

Studies included had to meet the following criteria: (1) case-control studies; (2) patients with essential hypertension were defined as cases, healthy subjects without hypertension were defined as controls; (3) evaluation of the association between eNOS rs1799983 polymorphism and hypertension. The exclusion criteria satisfied the followings: (1) case reports, review articles or cross-sectional studies; (2) duplicate articles; (3) secondary hypertension or gestational hypertension; (4) lack of sufficient information on genotype or allele frequencies.

\section{Data extraction and quality assessment}

For each eligible study, the following data were extracted: name of first author, year of publication, region and ethnicity of study population, sample size, and numbers of eNOS genotype or allele in cases and controls. Hardy-Weinberg equilibrium (HWE) among the controls was calculated.

Quality of the included studies was evaluated by two investigators (Jikang Shi and Yanbo Guo) independently using the Newcastle-Ottawa scale (NOS) [14] that has a "star" rating system consisting of selection, comparability, and exposure. The highest score of this rating system is 9 points. Inconformity was settled by discussing with the third investigator (Sainan Liu) if the evaluation results of two investigators didn't reach an agreement. 


\section{Statistical analysis}

HWE was evaluated for control groups of each study using Goodness of fit Chi-square test, and $P<0.05$ was considered as a significant deviation from HWE. The associations between eNOS rs1799983 polymorphisms and hypertension in this meta-analysis were measured based on five different genetic models including six comparisons: allelic model (T vs G), codominant model (GT vs GG and TT vs GG), dominant model (GT + TT vs GG), recessive model (TT vs GG $+\mathrm{GT}$ ), overdominant model (GT vs GG + TT). Odds ratios $(O R)$ and $95 \%$ confidence intervals $(95 \% \mathrm{Cl})$ were used to assess the strength of association between eNOS rs 1799983 polymorphisms and hypertension. Q-statistic and $R^{2}$-statistic were used to evaluate heterogeneity, random-effect models (DerSimonian and Laird methods) were used when heterogeneity existed ( $R \geq 50 \%$ considered heterogeneity existed in between-study in this meta-analysis); otherwise, fixedeffect models (Mantel and Haenszel methods) were used. Subgroup analyses were performed by region, ethnicity, and HWE to detect main sources of heterogeneity and observe differences of the association in different groups. Sensitivity analysis was conducted to evaluate stability of our results by omitting each study at each time. Publication bias was estimated using funnel plots, and quantified by the Egger's tests $(P<0.05$ considered statistically significant publication bias) [15]. All data management and statistical analyses were performed using R Studio (Version 1.1.383) (RStudio, Inc., MA, USA) for Windows.

\section{Trial sequential analysis (TSA)}

The risk of random error in traditional meta-analysis may increase because of the dispersed data and repeated significance testing [16, 17]. TSA was used to reduce the risk of type I error by adjusting threshold for statistical significance and to evaluate the required information size (RIS) and statistical reliability [18]. In our meta-analysis, trial sequential analysis software (TSA, version 0.9; Copenhagen Trial Unit, Copenhagen, Denmark, 2011) were performed, and additional studies were not needed when Z-curve crossed the trial sequential monitoring boundary or RIS has reached; otherwise, further studies were needed.

\section{Results}

\section{Study Characteristics}

A total of 60 eligible articles involving 14,185 cases and 13,407 controls were finally selected after strict screening on the basis of the inclusion and exclusion criteria, the protocol of literature search and selection is shown in Fig. 1, and the main characteristics and genotype distribution of the eligible studies are listed in Table 1. 
Table 1

Main characteristics of the included studies.

\begin{tabular}{|c|c|c|c|c|c|c|c|c|c|c|c|c|c|}
\hline \multirow{3}{*}{$\begin{array}{l}\text { Study } \\
\text { Lacolley }\end{array}$} & \multirow{3}{*}{$\begin{array}{l}\text { Year } \\
1997 \\
\end{array}$} & \multirow{3}{*}{$\begin{array}{l}\text { Region } \\
\text { France }\end{array}$} & \multirow{3}{*}{$\begin{array}{l}\text { Ethnicity } \\
\text { Caucasian }\end{array}$} & \multirow{2}{*}{\multicolumn{2}{|c|}{$\begin{array}{l}\text { Sample size } \\
\text { (case/control) }\end{array}$}} & \multirow{3}{*}{$\begin{array}{l}\text { Quality } \\
\text { score } \\
7\end{array}$} & \multirow{3}{*}{$\begin{array}{l}\text { HWE } \\
Y / N \\
0.250\end{array}$} & \multicolumn{2}{|c|}{ GG (n) } & \multicolumn{2}{|c|}{ GT (n) } & \multicolumn{2}{|c|}{ TT (n) } \\
\hline & & & & & & & & case & control & case & control & case & control \\
\hline & & & & $309 /$ & 123 & & & 140 & 35 & 122 & 67 & 47 & 21 \\
\hline Miyamoto & 1998 & Japan & Asian & $218 /$ & 240 & 8 & 0.587 & 175 & 217 & 41 & 22 & 2 & 1 \\
\hline Benjafield & 2000 & Australia & Caucasian & $91 /$ & 149 & 7 & 0.314 & 40 & 70 & 43 & 68 & 8 & 11 \\
\hline Shoji & 2000 & Japan & Asian & $183 /$ & 193 & 7 & 0.462 & 139 & 164 & 41 & 27 & 3 & 2 \\
\hline KARVONEN & 2002 & Finland & Caucasian & $505 /$ & 519 & 9 & 0.820 & 244 & 262 & 220 & 215 & 41 & 42 \\
\hline Di & 2002 & China & Asian & $95 /$ & 95 & 7 & 0.511 & 70 & 83 & 25 & 12 & 0 & 0 \\
\hline Liu & 2002 & China & Asian & $103 /$ & 74 & 7 & 0.205 & 54 & 55 & 44 & 19 & 5 & 0 \\
\hline Jia & 2002 & China & Asian & $116 /$ & 136 & 8 & 0.316 & 83 & 114 & 29 & 20 & 4 & 2 \\
\hline Tan & 2003 & China & Asian & $112 /$ & 112 & 8 & 0.012 & 73 & 78 & 25 & 26 & 14 & 8 \\
\hline $\mathrm{Li}$ & 2004 & China & Asian & $310 /$ & 151 & 8 & 0.902 & 226 & 126 & 81 & 24 & 3 & 1 \\
\hline $\mathrm{Xu}$ & 2004 & China & Asian & $203 /$ & 190 & 8 & 0.854 & 165 & 141 & 37 & 45 & 1 & 4 \\
\hline Djuric' & 2005 & Serbia & Caucasian & $172 /$ & 200 & 7 & 0.782 & 84 & 93 & 71 & 88 & 17 & 19 \\
\hline Moe & 2006 & Singapore & Asian & $103 /$ & 104 & 7 & 0.787 & 79 & 82 & 20 & 21 & 4 & 1 \\
\hline Marcun-Varda & 2006 & Slovenia & Caucasian & $104 /$ & 200 & 7 & 0.901 & 43 & 74 & 49 & 96 & 12 & 30 \\
\hline Dong & 2006 & China & Asian & $97 /$ & 87 & 7 & 0.983 & 41 & 62 & 50 & 23 & 6 & 2 \\
\hline $\mathrm{Ma}$ & 2006 & China & Asian & $192 /$ & 122 & 7 & 0.274 & 76 & 46 & 89 & 53 & 27 & 23 \\
\hline Wang & 2006 & China & Asian & $277 /$ & 547 & 7 & 0.284 & 233 & 468 & 40 & 74 & 4 & 5 \\
\hline Zhang & 2006 & China & Asian & $375 /$ & 414 & 7 & $<0.001$ & 212 & 273 & 106 & 93 & 57 & 48 \\
\hline Liang & 2006 & China & Asian & $124 /$ & 100 & 8 & 0.625 & 108 & 85 & 11 & 14 & 5 & 1 \\
\hline Zhang & 2006 & China & Asian & $190 /$ & 94 & 8 & 0.791 & 164 & 89 & 19 & 5 & 7 & 0 \\
\hline Zhao & 2006 & China & Asian & $501 /$ & 489 & 7 & 0.692 & 404 & 387 & 93 & 97 & 4 & 5 \\
\hline Khawaja & 2007 & Pakistan & Mixed & $143 /$ & 184 & 6 & 0.689 & 99 & 129 & 37 & 51 & 7 & 4 \\
\hline Wang & 2007 & China & Asian & $100 /$ & 50 & 7 & 0.101 & 70 & 44 & 27 & 5 & 3 & 1 \\
\hline Colomba & 2008 & Italy & Caucasian & $127 /$ & 67 & 7 & 0.030 & 45 & 19 & 70 & 41 & 12 & 7 \\
\hline Nejatizadeh & 2008 & India & Asian & $453 /$ & 344 & 7 & 0.006 & 259 & 222 & 118 & 98 & 76 & 24 \\
\hline Periaswamy & 2008 & India & Asian & $438 /$ & 444 & 8 & 0.656 & 291 & 323 & 126 & 110 & 21 & 11 \\
\hline Srivastava & 2008 & India & Asian & $226 /$ & 200 & 8 & 0.556 & 139 & 154 & 82 & 44 & 5 & 2 \\
\hline Ghazali & 2008 & Malaysia & Asian & $200 /$ & 198 & 8 & 0.920 & 144 & 151 & 54 & 44 & 2 & 3 \\
\hline Tang & 2008 & China & Asian & $184 /$ & 196 & 6 & 0.983 & 91 & 95 & 80 & 83 & 13 & 18 \\
\hline Zhao & 2008 & China & Asian & $174 /$ & 112 & 7 & 0.733 & 138 & 105 & 32 & 7 & 4 & 0 \\
\hline Tang & 2008 & China & Asian & $271 /$ & 267 & 6 & $<0.001$ & 171 & 169 & 73 & 65 & 27 & 33 \\
\hline Wang & 2009 & China & Asian & $230 /$ & 186 & 8 & 0.518 & 9 & 12 & 46 & 64 & 175 & 110 \\
\hline Zhang & 2009 & China & Asian & $349 /$ & 214 & 8 & 0.267 & 260 & 179 & 79 & 32 & 10 & 3 \\
\hline Liu & 2009 & China & Asian & $129 /$ & 117 & 7 & 0.311 & 76 & 85 & 46 & 31 & 7 & 1 \\
\hline Niu & 2009 & China & Asian & $1305 /$ & 1154 & 8 & 0.008 & 1071 & 954 & 192 & 182 & 42 & 18 \\
\hline Kitsios & 2010 & Greece & Caucasian & $228 /$ & 302 & 6 & 0.512 & 99 & 135 & 95 & 130 & 34 & 37 \\
\hline Wang & 2010 & China & Asian & $154 /$ & 150 & 8 & 0.240 & 98 & 116 & 40 & 30 & 16 & 4 \\
\hline Zhou & 2010 & China & Asian & $176 /$ & 131 & 6 & 0.351 & 137 & 98 & 38 & 32 & 1 & 1 \\
\hline Souza-Costa & 2011 & Brazil & Mixed & $73 /$ & 285 & 8 & 0.086 & 45 & 172 & 25 & 105 & 3 & 8 \\
\hline Zhou & 2011 & China & Asian & $346 /$ & 385 & 8 & 0.667 & 280 & 312 & 62 & 70 & 4 & 3 \\
\hline
\end{tabular}




\begin{tabular}{|c|c|c|c|c|c|c|c|c|c|c|c|c|c|}
\hline \multirow{2}{*}{$\begin{array}{l}\text { Study } \\
\text { Chen }\end{array}$} & \multirow{2}{*}{$\begin{array}{l}\text { Year } \\
2011\end{array}$} & \multirow{2}{*}{$\begin{array}{l}\text { Region } \\
\text { China }\end{array}$} & \multirow{2}{*}{$\begin{array}{l}\text { Ethnicity } \\
\text { Asian }\end{array}$} & \multicolumn{2}{|c|}{ Sample size } & \multirow{2}{*}{$\begin{array}{l}\text { Quality } \\
8\end{array}$} & \multirow{2}{*}{$\begin{array}{l}\text { HWE } \\
0.161\end{array}$} & \multicolumn{2}{|c|}{ GG (n) } & \multicolumn{2}{|c|}{ GT (n) } & \multicolumn{2}{|c|}{ TT (n) } \\
\hline & & & & $160 /$ & 176 & & & 138 & 154 & 21 & 20 & 1 & 2 \\
\hline Zhao & 2011 & China & Asian & $100 /$ & 97 & 8 & 0.648 & 96 & 82 & 3 & 14 & 1 & 1 \\
\hline $\mathrm{Li}$ & 2011 & China & Asian & $510 /$ & 510 & 7 & $<0.001$ & 320 & 367 & 129 & 89 & 61 & 54 \\
\hline $\mathrm{Ma}$ & 2012 & China & Asian & $300 /$ & 288 & 8 & 0.577 & 255 & 250 & 43 & 36 & 2 & 2 \\
\hline Zhang & 2012 & China & Asian & $363 /$ & 370 & 6 & 0.580 & 265 & 278 & 85 & 84 & 13 & 8 \\
\hline Liang & 2012 & China & Asian & $350 /$ & 150 & 7 & 0.965 & 290 & 127 & 57 & 22 & 3 & 1 \\
\hline $\mathrm{Li}$ & 2012 & China & Asian & $227 /$ & 359 & 7 & 0.549 & 185 & 296 & 40 & 61 & 2 & 2 \\
\hline Goncharov & 2013 & Ukraine & Caucasian & $145 /$ & 144 & 7 & $<0.001$ & 65 & 45 & 60 & 93 & 20 & 6 \\
\hline Yan & 2013 & China & Asian & $308 /$ & 181 & 8 & 0.105 & 235 & 142 & 57 & 34 & 16 & 5 \\
\hline Yang & 2013 & China & Asian & 134/ & 115 & 6 & 0.791 & 70 & 97 & 59 & 17 & 5 & 1 \\
\hline Ogretmen & 2014 & Turkey & Caucasian & $21 /$ & 109 & 6 & 0.746 & 7 & 70 & 13 & 34 & 1 & 5 \\
\hline Shankarishan & 2014 & India & Caucasian & 350 & $/ 350$ & 8 & 0.261 & 194 & 296 & 133 & 50 & 23 & 4 \\
\hline Cui & 2014 & China & Asian & 172 & $/ 90$ & 8 & 0.786 & 133 & 85 & 36 & 5 & 3 & 0 \\
\hline Liu & 2014 & China & Asian & 215 & $/ 108$ & 8 & 0.283 & 149 & 89 & 48 & 17 & 18 & 2 \\
\hline Hui & 2015 & China & Asian & 100 & $/ 100$ & 6 & 0.677 & 81 & 92 & 16 & 8 & 3 & 0 \\
\hline Xiong & 2015 & China & Asian & 226 & $/ 186$ & 8 & 0.752 & 130 & 133 & 83 & 48 & 13 & 5 \\
\hline ALrefai & 2016 & Egypt & Caucasian & 70 & $/ 30$ & 7 & 0.773 & 49 & 27 & 16 & 3 & 5 & 0 \\
\hline Gamil & 2017 & Sudan & Caucasian & 147 & /82 & 6 & 0.829 & 100 & 60 & 42 & 20 & 5 & 2 \\
\hline Zhang & 2017 & China & Asian & 456 & $/ 453$ & 8 & 0.001 & 365 & 362 & 84 & 78 & 7 & 13 \\
\hline Nassereddine & 2018 & Morocco & Caucasian & 145 & $/ 184$ & 6 & 0.509 & 5 & 116 & 54 & 62 & 86 & 6 \\
\hline
\end{tabular}

\section{Association between eNOS rs1799983 polymorphism and hypertension}

There were significant heterogeneities between eNOS rs1799983 polymorphism and hypertension in the five different genetic models, and thus random-effects model was used for all analyses. We found significant association between eNOS rs1799983 polymorphism and the risk of hypertension under any genetic model ( $T$ vs $G: O R=1.44,95 \% C l=1.26-1.63$; $G T$ vs $G G: O R=1.34,95 \% C l=1.18-1.52$; TT vs GG: $O R=1.80,95 \% C l=1.41-2.31 ; \mathrm{GT}+\mathrm{TT}$ vs GG: $O R=1.42$, $95 \% C l=1.25-1.63 ; \mathrm{TT}$ vs GG + GT: $O R=1.68,95 \% C l=1.35-2.08 ; \mathrm{GT}$ vs GG + TT: $O R=1.24,95 \% C l=1.11-1.40)($ Fig. 2).

\section{Subgroup analysis}

We performed subgroup analysis by region and ethnicity because gene polymorphism may be associated with variations in region and ethnicity. For region, there is only difference for the association between eNOS rs1799983 polymorphism and hypertension under overdominant model, when GT was compared with GG + TT, the association with risk of hypertension was identified in China (OR=1.29;95\% Cl=1.12-1.49), and the association between eNOS rs 1799983 polymorphism with risk of hypertension was found in any region under other genetic models. With regard to ethnicity, we found the association between eNOS rs1799983 polymorphism with risk of hypertension was significant in Asian population under all genetic models (T vs $\mathrm{G}$ : $O R=1.42,95 \% \mathrm{Cl}=1.27-1.58 ; \mathrm{GT}$ vs GG: $O R=1.37,95 \% C l=1.21-1.54 ; \mathrm{TT}$ vs GG: $O R=1.64,95 \% C l=1.35-2.00 ; \mathrm{GT}+\mathrm{TT}$ vs GG: $O R=1.43,95 \% C l=1.27-1.61 ; \mathrm{TT}$ vs GG + GT:OR=1.56,95\% $C l=$ 1.29-1.88; GT vs GG + TT: $O R=1.31,95 \% C l=1.15-1.48$ ); however, with respect to contrast of TT versus GG and TT versus $G G+G T$, the genotype TT was associated with the increased risk of hypertension not only in Asian population but also in other population $(O R=2.07,95 \% C l=1.05-4.08$ and $O R=1.87$, $95 \% C l=1.07-3.25$, respectively) (Table 2 ). 
Table 2

Overall and subgroup analysis of association between eNOS rs1799983 polymorphism and hypertension under different models.

\begin{tabular}{|c|c|c|c|c|c|c|c|c|c|c|c|c|c|c|c|}
\hline \multirow[t]{2}{*}{ Categories } & \multicolumn{3}{|c|}{ T vs G } & \multicolumn{3}{|c|}{ GT vs GG } & \multicolumn{3}{|c|}{ TT vs GG } & \multicolumn{3}{|c|}{ GT + TT vs GG } & \multicolumn{3}{|c|}{ TT vs $G G+G T$} \\
\hline & $O R$ & $(95 \% \mathrm{Cl})$ & $\begin{array}{l}R \\
(\%)\end{array}$ & $O R$ & $(95 \% \mathrm{Cl})$ & $\begin{array}{l}R \\
(\%)\end{array}$ & $O R$ & $(95 \% \mathrm{Cl})$ & $\begin{array}{l}R \\
(\%)\end{array}$ & $O R$ & $(95 \% \mathrm{Cl})$ & $\begin{array}{l}R \\
(\%)\end{array}$ & $O R$ & $(95 \% \mathrm{Cl})$ & $\begin{array}{l}R \\
(\%)\end{array}$ \\
\hline Overall & 1.44 & $(1.26,1.63)$ & 85 & 1.34 & $(1.18,1.52)$ & 75 & 1.80 & $(1.41,2.31)$ & 65 & 1.42 & $(1.25,1.63)$ & 79 & 1.68 & $(1.35,2.08)$ & 58 \\
\hline \multicolumn{16}{|l|}{ Region } \\
\hline China & 1.40 & $(1.23,1.59)$ & 72 & 1.35 & $(1.18,1.55)$ & 65 & 1.54 & $(1.24,1.93)$ & 24 & 1.42 & $(1.23,1.63)$ & 69 & 1.47 & $(1.19,1.81)$ & 24 \\
\hline Other & 1.47 & $(1.12,1.91)$ & 92 & 1.31 & $(1.01,1.71)$ & 85 & 2.05 & $(1.24,3.40)$ & 82 & 1.44 & $(1.09,1.89)$ & 87 & 1.89 & $(1.24,2.88)$ & 77 \\
\hline \multicolumn{16}{|l|}{ Ethnicity } \\
\hline Asian & 1.42 & $(1.27,1.58)$ & 69 & 1.37 & $(1.21,1.54)$ & 63 & 1.64 & $(1.35,2.00)$ & 23 & 1.43 & $(1.27,1.61)$ & 66 & 1.56 & $(1.29,1.88)$ & 23 \\
\hline Other & 1.44 & $(0.98,2.12)$ & 94 & 1.28 & $(0.87,1.87)$ & 88 & 2.07 & $(1.05,4.08)$ & 88 & 1.42 & $(0.94,2.15)$ & 91 & 1.87 & $(1.07,3.25)$ & 83 \\
\hline
\end{tabular}

\section{Sensitivity analysis and publication bias}

To examine the influence of individual study on the overall results, sensitivity analysis was performed by excluding a single study at each time in our metaanalysis. The results of sensitivity analysis showed that the corresponding pooled ORs and $95 \% \mathrm{Cls}$ under any model of inheritance were not substantially altered after excluding any single study, suggesting that results of our meta-analysis were relatively stable and credible (Fig. 3).

Publication bias was evaluated by funnel plots and quantified by Egger's tests. The funnel plots for recessive model (TT vs GG + GT) seemed symmetrical, and the results of Egger's tests showed that there was no publication bias $(P=0.102)$; however, the funnel plots were asymmetrical in other genetic models for the association between eNOS rs1799983 polymorphism with hypertension, and the results of Egger's tests showed that there were publication bias (T vs G: $P=$ 0.026; GT vs GG: $P=0.023$; TT vs GG: $P=0.032 ; \mathrm{GT}+\mathrm{TT}$ vs GG: $P=0.011 ; \mathrm{GT}$ vs GG + TT: $P=0.038$ ) (Fig. 4).

\section{Trial sequential analysis (TSA)}

For the association between eNOS rs1799983 polymorphism with hypertension under codominant model (GT vs GG), codominant model (TT vs GG), and dominant model (GT + TT vs GG), the Z-curve crossed trial sequential monitoring boundary, although the sample size did not reach the RIS (Fig. 5B, 5C, and 5D). However, for the association between eNOS rs 1799983 polymorphism with hypertension under allelic model (T vs G), recessive model (TT vs GG + GT), and overdominant model (GT vs GG + TT), the Z-curve crossed trial sequential monitoring boundary, and the sample sizes were also more than the RIS (Fig. 5A, 5E, and 5F). Therefore, concrete evidence indicates that further studies are not necessary for the association between eNOS rs1799983 polymorphism with hypertension.

\section{Discussion}

In the meta-analysis, we collected related articles comprehensively to investigate the association between eNOS rs 1799983 polymorphism and hypertension. Our results show that an association between eNOS rs 1799983 polymorphism and risk of hypertension is identified under any genetic model ( $T$ vs G, GT vs GG, TT vs GG, GT + TT vs GG, TT vs GG + GT, and GT vs GG + TT), especially among Asian population. Moreover, with respect to contrast of TT versus GG and TT versus $G G+G T$, the TT genotype is associated with the increased risk of hypertension not only in Asian population but also in other population.

Nine meta-analyses on association between eNOS rs1799983 polymorphism and hypertension have been published, four of them (Chen et al., Wang et al., Li et al., and Liu et al) studied the Chinese populations[19-22]. Chen et al. and Wang et al. studied the two models (T vs G and GT + TT vs GG) of our models in this meta-analysis, and their results are consistent with our results, we all found that T allele and GT + TT genotype are associated with an increased risk of hypertension. In addition, Li et al. studied the association between T allele of eNOS rs1799983 polymorphism and hypertension, and Liu et al studied the association between GT + TT genotype of eNOS rs1799983 polymorphism and hypertension, and their results are also consistent with our results.

Pereira et al [23] studied the association between GT + TT genotype of eNOS rs1799983 polymorphism and hypertension, and consistent with the discoveries of Pereira et al, we also identified the heterogeneity and publication bias in the meta-analysis, they may exist owing to the gene-environment interactions. Niu [24] et al only studied the association between T allele of eNOS rs1799983 polymorphism and hypertension, we all found the T allele of eNOS rs 1799983 polymorphism was risk factor of hypertension, especially among Asian population. Moreover, of the nine meta-analyses, the results of Takeuchi [25] and Zintzaras [26] were negative, they found that there was no association between eNOS rs1799983 polymorphism and hypertension, the reason they had this inconsistency in results may be a small size, or interaction of polymorphisms within haplotypes, which is a major determinant of disease susceptibility, not the individual polymorphism[27].

For the meta-analysis of Xie [13] et al, the last meta-analysis published in 2017, their results showed there is no association between TT genotype and hypertension when TT genotype was compared with GG + GT genotype, but TT genotype was associated with the increased risk of hypertension in our metaanalysis. In addition, our result of TSA demonstrated that the Z-curve crossed trial sequential monitoring boundary, and the sample sizes were also more than the RIS. Therefore, it is adequate to draw a conclusion that TT genotype is associated with the increased risk of hypertension.

The meta-analysis may report false positive results for the risk of type I errors, and these results are usually due to publication bias, heterogeneity between studies, and poor study quality. However, a limited number of trials may not provide enough information, resulting in incorrect estimates [28]. Thus, we 
conducted TSA to reduce the risk of type I errors and evaluated whether further studies are necessary by calculating the required information size. In our metaanalysis, either the sample size was greater than the required information size or the Z-curve crossed trial sequential monitoring boundary, indicating that the results of our meta-analysis are reliable and sufficient to draw conclusions on the association between eNOS rs1799983 polymorphism and hypertension.

Our study has some limitations. First, there is heterogeneity in our article, and the main sources of heterogeneity remain unclear. Second, publication bias was found in the association between eNOS rs1799983 polymorphism and hypertension under any genetic model except the recessive model, because negative articles are unpublished. Third, our research cannot prove the existence of causality, but only an association because of the design of case-control.

Despite the above limitations, our research also has some advantages. First of all, we have collected the latest articles extensively, which provides more statistical power to draw effective conclusions on this issue. Secondly, the results of sensitivity analysis show that our conclusion is stable and reliable. Third, as far as we know, this is the first TSA to evaluate the association between eNOS rs1799983 polymorphism and hypertension, which further gives reliable evidence to reach the conclusion.

\section{Conclusion}

In conclusion, eNOS rs1799983 polymorphism is associated with increased risk of hypertension under any genetic model. Moreover, investigations of genegene and gene-environment interactions are needed to give more insight into the association between eNOS rs1799983 polymorphism and hypertension.

\section{Abbreviations}

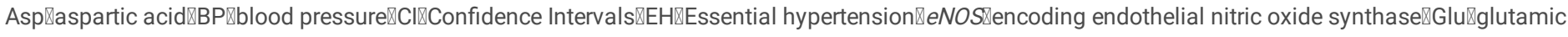

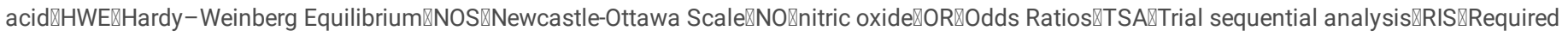
Information Size

\section{Declarations}

\section{Acknowledgements}

We thank all the participants of the study.

\section{Funding}

This work was supported by the funds from National Natural Science Foundation of China (81973120), National Key R\&D Program of China (2018YFC1311600), and Graduate Innovation Fund of Jilin University (101832020CX267).

\section{Availability of data and materials}

All data generated or analysed during this study are included in this published article.

\section{Author Contributions}

Conception and design: Jikang Shi, Yi Cheng, and Yawen Liu. Provision of study materials: Jikang Shi, Siyu Liu, Yanbo Guo, and Sainan Liu. Collection and assembly of data: Jikang Shi, Jiayi Xu, Lingfeng Pan, and Yueyang Hu. Data analysis and interpretation: Jikang Shi and Siyu Liu. Manuscript writing: Jikang Shi. Revised the language/article: All authors. Final approval of manuscript: All authors.

\section{Ethics approval and consent to participate}

Not applicable.

\section{Consent for publication}

Not applicable.

\section{Conflict of interest}

The authors declare no conflict of interest.

\section{References}


1. Pierdomenico SD, Di Nicola M, Esposito AL, Di Mascio R, Ballone E, Lapenna D, et al. Prognostic value of different indices of blood pressure variability in hypertensive patients. Am J Hypertens. 2009; 22:842-7.

2. Hong Y, de Faire U, Heller DA, McClearn GE, Pedersen N. Genetic and environmental influences on blood pressure in elderly twins. Hypertension. 1994; 24:663-70.

3. Niu WQ, Qi Y. Meta-based evidence for apolipoprotein E epsilon2/epsilon3/epsilon4 polymorphism in association with hypertension among Chinese. J Hum Hypertens. 2011; 25:725-31.

4. Zhuo ML, Huang Y, Chen JZ, Sun LH, Yang RF, Chen HZ, et al. Endothelium-specific overexpression of human IC53 downregulates endothelial nitric oxide synthase activity and elevates systolic blood pressure in mice. Cardiovasc Res. 2009; 84:292-9.

5. Ohashi Y, Kawashima S, Hirata K, Yamashita T, Ishida T, Inoue N, et al. Hypotension and reduced nitric oxide-elicited vasorelaxation in transgenic mice overexpressing endothelial nitric oxide synthase. J Clin Invest. 1998; 102:2061-71.

6. Gamboa A, Shibao C, Diedrich A, Choi L, Pohar B, Jordan J, et al. Contribution of endothelial nitric oxide to blood pressure in humans. Hypertension. 2007; 49:170-7.

7. Hingorani AD, Liang CF, Fatibene J, Lyon A, Monteith S, Parsons A, et al. A common variant of the endothelial nitric oxide synthase (Glu298->Asp) is a major risk factor for coronary artery disease in the UK. Circulation. 1999; 100:1515-20.

8. Ong SL, Whitworth JA. How do glucocorticoids cause hypertension: role of nitric oxide deficiency, oxidative stress, and eicosanoids. Endocrinol Metab Clin North Am. 2011; 40:393-407, ix.

9. AA AL, Habib MS, Yaseen RI, Gabr MK, Habeeb RM. Association of endothelial nitric oxide synthase (eNOS) gene G894T polymorphism with hypertension risk and complications. Mol Cell Biochem. 2016; 421:103-10.

10. Gamil S, Erdmann J, Abdalrahman IB, Mohamed AO. Association of NOS3 gene polymorphisms with essential hypertension in Sudanese patients: a case control study. BMC Med Genet. 2017; 18:128.

11. Zhang W: Correlation between eNOS gene polymorphism and essential hypertension in Guizhou Han, Miao and Buyi. Master. Guizhou Medical University, 2017.

12. Nassereddine S, Hassani Idrissi H, Habbal R, Abouelfath R, Korch F, Haraka M, et al. The polymorphism G894 T of endothelial nitric oxide synthase (eNOS) gene is associated with susceptibility to essential hypertension (EH) in Morocco. BMC Med Genet. 2018; 19:127.

13. Xie X, Shi X, Xun X, Rao L. Endothelial nitric oxide synthase gene single nucleotide polymorphisms and the risk of hypertension: A meta-analysis involving 63,258 subjects. Clin Exp Hypertens. 2017; 39:175-82.

14. Stang A. Critical evaluation of the Newcastle-Ottawa scale for the assessment of the quality of nonrandomized studies in meta-analyses. Eur $\mathrm{J}$ Epidemiol. 2010; 25:603-5.

15. Begg CB, Mazumdar M. Operating characteristics of a rank correlation test for publication bias. Biometrics. 1994; 50:1088-101.

16. Turner RM, Bird SM, Higgins JP. The impact of study size on meta-analyses: examination of underpowered studies in Cochrane reviews. PLoS One. 2013; 8:e59202.

17. Brok J, Thorlund K, Wetterslev J, Gluud C. Apparently conclusive meta-analyses may be inconclusive--Trial sequential analysis adjustment of random error risk due to repetitive testing of accumulating data in apparently conclusive neonatal meta-analyses. Int J Epidemiol. 2009; 38:287-98.

18. Imberger G, Gluud C, Wetterslev J. Comments on 'Sequential methods for random-effects meta-analysis' by J. P. Higgins, A. Whitehead and M. Simmonds, Statistics in Medicine 2010; DOI: 10.1002/sim.4088. Stat Med. 2011; 30:2965-6.

19. Chen J, Zhou Z, Ke J, Chen X. Meta-analysis of the relationship between endothelial nitric oxide synthase gene G894T polymorphism and essential hypertension,. Journal of Sun Yat-sen University (Medical Science Edition). 2008:116-19.

20. Wang C, Zhao J, Xu J, Xiang Z, Liang C, Li J. Meta-analysis of the relationship between G894T polymorphism of Chinese endothelial nitric oxide synthase gene and essential hypertension. Chinese Journal of Epidemiology. 2009; 30:845-49.

21. Li YY. Endothelial nitric oxide synthase G894T gene polymorphism and essential hypertension in the Chinese population: a meta-analysis involving 11,248 subjects. Intern Med. 2011; 50:2099-106.

22. Liu J, Wang L, Liu Y, Wang Z, Li M, Zhang B, et al. The association between endothelial nitric oxide synthase gene G894T polymorphism and hypertension in Han Chinese: a case-control study and an updated meta-analysis. Ann Hum Biol. 2015; 42:184-94.

23. Pereira TV, Rudnicki M, Cheung BM, Baum L, Yamada Y, Oliveira PS, et al. Three endothelial nitric oxide (NOS3) gene polymorphisms in hypertensive and normotensive individuals: meta-analysis of 53 studies reveals evidence of publication bias. J Hypertens. 2007; 25:1763-74.

24. Niu W, Qi Y. An updated meta-analysis of endothelial nitric oxide synthase gene: three well-characterized polymorphisms with hypertension. PLoS One. 2011; 6:e24266.

25. Takeuchi F, Yamamoto K, Katsuya T, Sugiyama T, Nabika T, Ohnaka K, et al. Reevaluation of the association of seven candidate genes with blood pressure and hypertension: a replication study and meta-analysis with a larger sample size. Hypertens Res. 2012; 35:825-31.

26. Zintzaras E, Kitsios G, Stefanidis I. Endothelial NO synthase gene polymorphisms and hypertension: a meta-analysis. Hypertension. 2006; 48:700-10.

27. Wang J, Dudley D, Wang XL. Haplotype-specific effects on endothelial NO synthase promoter efficiency: modifiable by cigarette smoking. Arterioscler Thromb Vasc Biol. 2002; 22:e1-4.

28. Thorlund K, Devereaux PJ, Wetterslev J, Guyatt G, loannidis JP, Thabane L, et al. Can trial sequential monitoring boundaries reduce spurious inferences from meta-analyses? Int J Epidemiol. 2009; 38:276-86. 


\section{Figures}

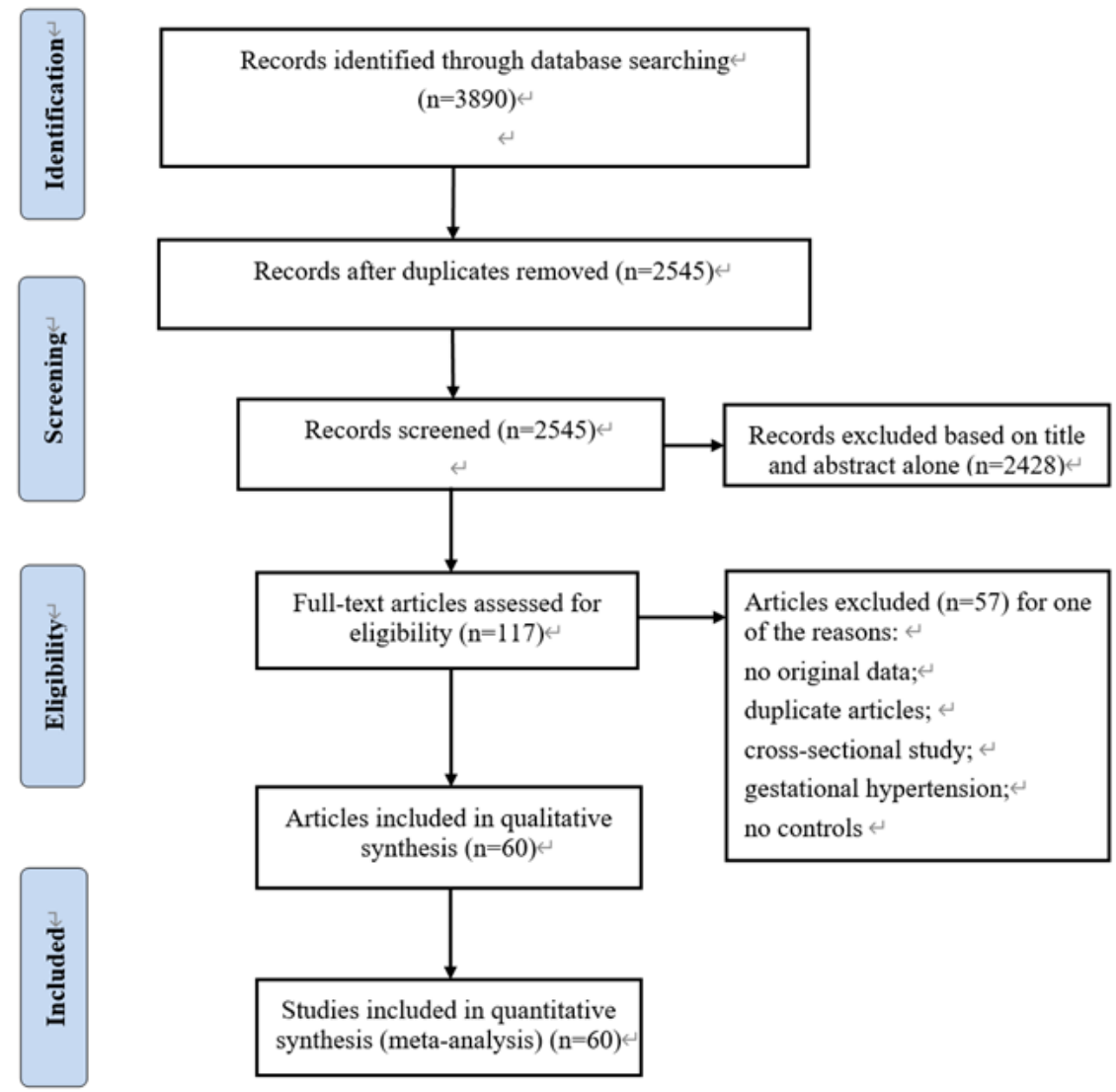

Figure 1

Flow chart of the process for literature identification and selection. 

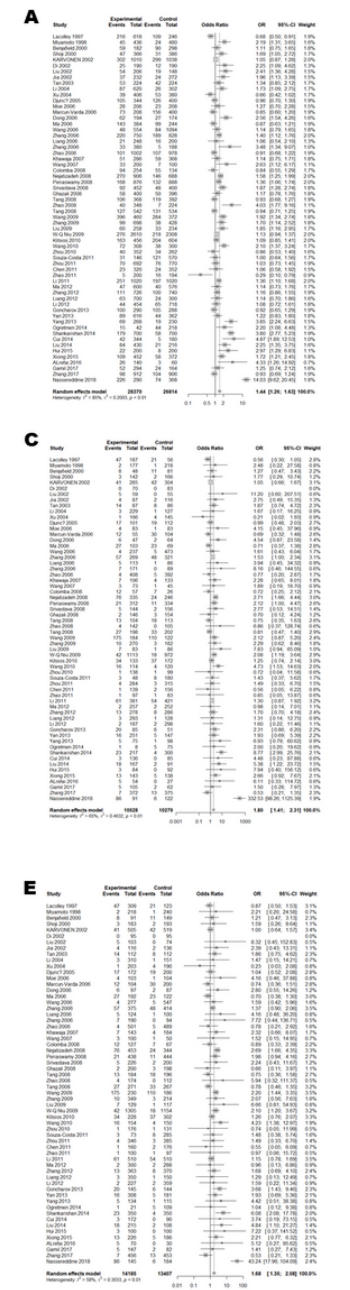
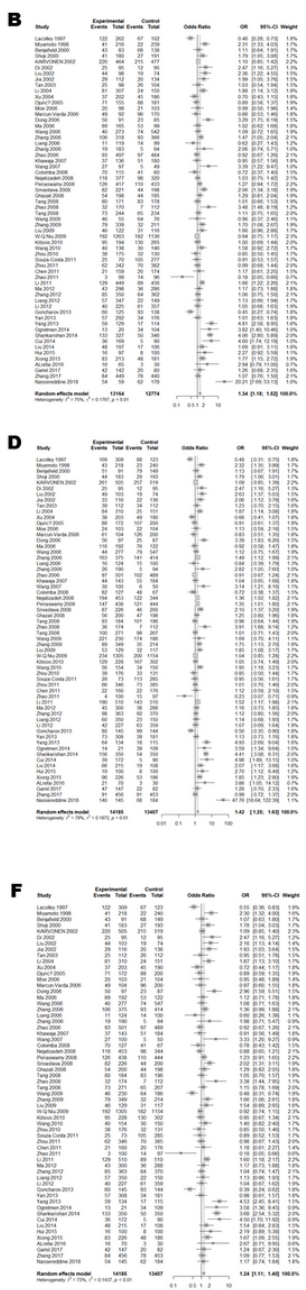

\section{Figure 2}

Forest plot for the result of association between eNOS rs1799983 polymorphism and hypertension based on a random-effects model. (A) allelic model: T vs G; (B) codominant model: GT vs GG; (C) codominant model: TT vs GG; (D) dominant model: GT+TT vs GG; (E) recessive model: TT vs GG+GT; (F) overdominant model: GT vs GG+TT 
A
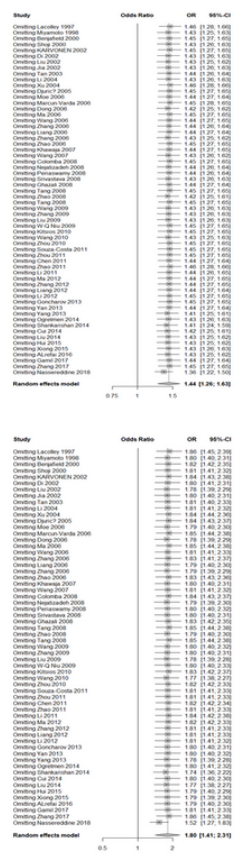

$\mathbf{E}$

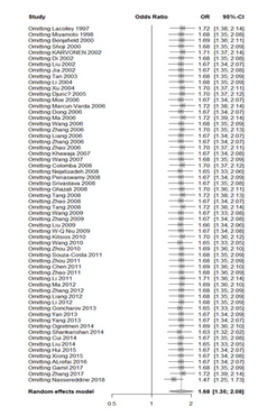

B

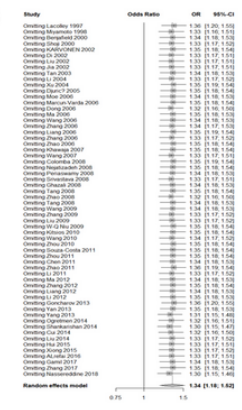

D

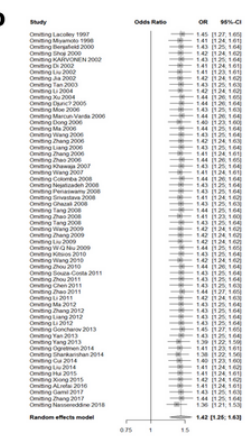

$\mathbf{F}$

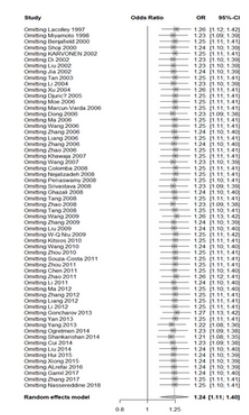

\section{Figure 3}

Sensitivity analysis of association between eNOS rs1799983 polymorphism and hypertension. (A) allelic model: T vs G; (B) codominant model: GT vs GG; (C) codominant model: TT vs GG; (D) dominant model: GT+TT vs GG; (E) recessive model: TT vs GG+GT; (F) overdominant model: GT vs GG+TT 
A

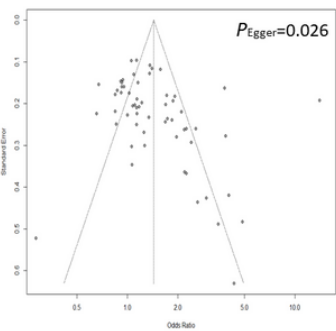

c

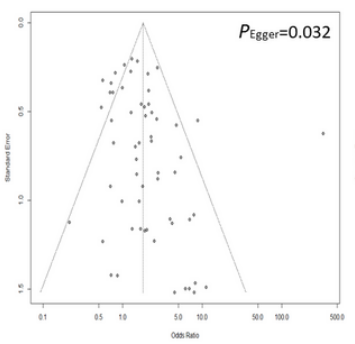

$\mathbf{E}$

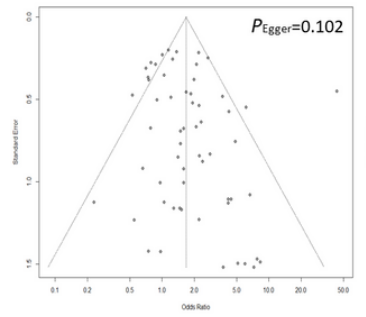

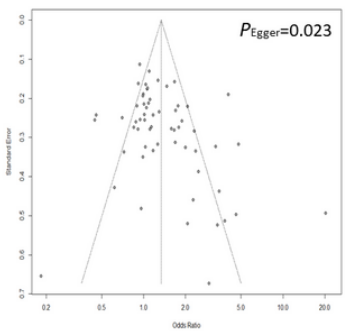

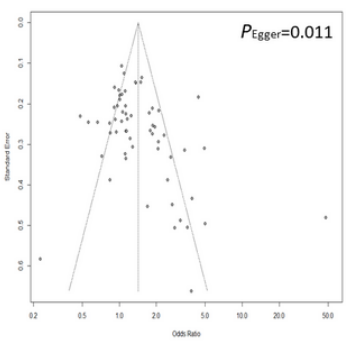

$\mathbf{F}$

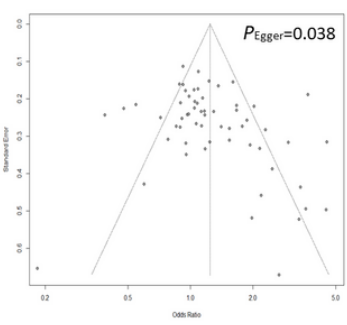

\section{Figure 4}

Funnel plot for the result of association between eNOS rs1799983 polymorphism and hypertension. (A) allelic model: T vs G; (B) codominant model: GT vs GG; (C) codominant model: TT vs GG; (D) dominant model: GT+TT vs GG; (E) recessive model: TT vs GG+GT; (F) overdominant model: GT vs GG+TT 

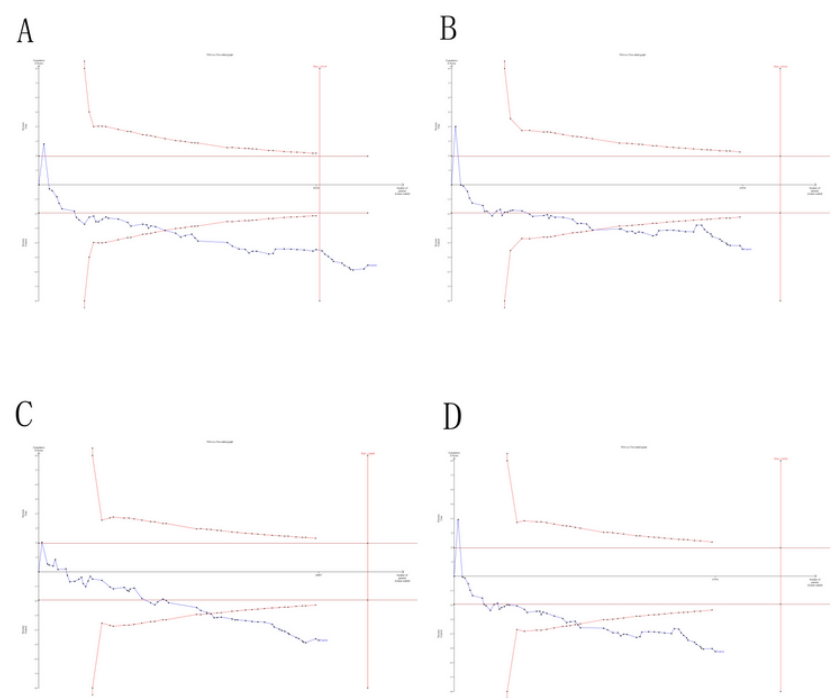

D
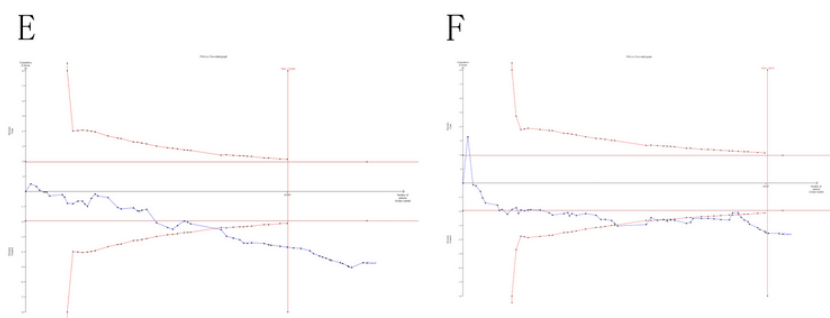

\section{Figure 5}

Trial sequential analysis of association between eNOS rs1799983 polymorphism and hypertension. (A) allelic model: T vs G; (B) codominant model: GT vs GG; (C) codominant model: TT vs GG; (D) dominant model: GT+TT vs GG; (E) recessive model: TT vs GG+GT; (F) overdominant model: GT vs GG+TT 